\title{
Aspectos clinicos de pacientes infanto-juvenis em terapia nutricional enteral domiciliar: uma revisão integrativa
}

\author{
I ${ }^{1}$ João Paulo Lima de Oliveira, ${ }^{2}$ Cynthia Francisca Xavier Costa de Assis Silva, \\ ${ }^{3}$ Lívia Garcia Ferreira, ${ }^{4}$ Lílian Gonçalves Teixeira I
}

Resumo: A Terapia Nutricional Enteral Domiciliar é um método eficaz para garantir o aporte nutricional de um indivíduo em seu domicílio. Sendo assim, o objetivo do estudo foi investigar os aspectos clínicos comumente recorrentes em pacientes pediátricos em terapia nutricional enteral domiciliar. A pesquisa de artigos foi realizada por meio das bases de dados: Scientific Eletronic Library Online (SciElo) e US National Library of Medicine National Institutes of Health (PubMed) utilizando as seguintes palavras-chaves: 'home enteral nutrition', 'home tube feeding' e 'children'.

Foram selecionados 16 estudos realizados nos países: Brasil, Chile, Espanha, França, Holanda, Itália, Polônia e Monte Sinai, os quais trouxeram evidências que as principais condiçóes clínicas relatadas para a indicação na terapia nutricional enteral domiciliar são as doenças neurológicas, neoplasias em geral e doenças do trato gastrointestinal. Apenas dois estudos abordaram os aspectos negativos da terapia domiciliar que são os episódios de engasgos, tosse, vômitos, diarreia e constipação intestinal. Deste modo, por meio das condiçóes clínicas relatadas observa-se que a indicação da TNED é essencial para estes pacientes, podendo preveni-los da desnutrição hospitalar, diminuição do tempo de permanência, reinternações e redução dos custos hospitalares, além de trazer maior conforto para o paciente pediátrico por estar mais próximo dos familiares.

> Palavras-chave: Terapia nutricional; Nutrição enteral; Visita domiciliar; Criança; Saúde da criança.

\author{
1 Universidade Federal de \\ Lavras. Lavras-MG, Brasil \\ (joaopaulolimanut@gmail.com). \\ ORCID: 0000-0002-9623-5474 \\ 2 Universidade Federal de Lavras \\ Lavras-MG, Brasil (cynthia.silva@ \\ ufla.br). \\ ORCID: 0000-0003-1110-7762 \\ ${ }^{3}$ Universidade Federal de Lavras \\ Lavras-MG, Brasil (livia.ferreira@ \\ ufla.br). \\ ORCID: 0000-0002-8089-351X \\ ${ }^{4}$ Universidade Federal de Lavras. \\ Lavras-MG, Brasil (lilian.teixeira@ \\ ufla.br). \\ ORCID: 0000-0003-4682-8594
}

Recebido em: 16/07/2019 Aprovado em: 13/01/2020 Revisado em: 18/05/2021 


\section{Introdução}

A hospitalização do paciente infanto-juvenil é um fator de risco de alta complexidade quanto ao desenvolvimento e exacerbação da desnutrição, comprometendo diretamente o estado nutricional das crianças e adolescentes. $\mathrm{O}$ mau prognóstico nutricional acarreta o aumento das taxas de morbimortalidade, longos períodos de internação e aumento dos custos hospitalares (PIMENTA et al., 2018).

A terapia nutricional é um método eficiente e requer abordagem individualizada, com orientação ou aconselhamento nutricional, educação alimentar e nutricional e intervenção dietética para indivíduos doentes. Durante a avaliação clínica, são coletados dados fisiopatológicos, situação clínica atual e pregressa, história social e familiar, avaliação antropométrica, bioquímica e dietética. Esses fatores serão primordiais para fornecer o diagnóstico nutricional e estabelecer a conduta nutricional adotada (RIBAS et al., 2016).

A Terapia Nutricional Enteral (TNE) é caracterizada por procedimentos terapêuticos com função de recuperar ou manter o estado nutricional dos indivíduos (WAITZBERG, 2009). A nutrição enteral é indicada para pacientes que não são capazes de suprir suas necessidades energéticas e nutricionais por via oral, entretanto, podem obter o aporte nutricional advindo de alguma parte do trato gastrointestinal que esteja com suas funcionalidades íntegras (SIDORKIEWICZ et al., 2012).

A Terapia Nutricional Domiciliar (TND) é definida como subsídio nutricional e clínico ao paciente em seu domicílio (SBNPE, 2012). Os primeiros serviços brasileiros de Atenção Domiciliar (AD) surgiram na década de 1960 como alternativa à internação hospitalar e em resposta à crise de lotação desses serviços. $\mathrm{Na}$ década de noventa, com o surgimento do Sistema Único de Saúde (SUS) e o processo de municipalização, esses serviços se multiplicaram, sendo regulamentados no SUS em 2002. Em 2006, a Agência Nacional de Vigilância Sanitária publicou um regulamento técnico de funcionamento dos serviços que prestam atençáo domiciliar (BRASIL, 2014a). Em 2011, foi criado o Programa Melhor em Casa, sistematizando a $\mathrm{AD}$ no SUS, vinculando-a à estratégia das redes de atenção à saúde (RAS) (BRASIL, 2016).

A Política Nacional de Alimentação e Nutrição (PNAN) tem como objetivo a melhoria das condiçôes de alimentação, nutrição e saúde da população brasileira, 
incluindo o cuidado integral dos agravos relacionados à alimentação e nutrição, onde se enquadram os indivíduos com necessidades alimentares especiais (BRASIL, 2013). A AD é parte do cuidado integral na RAS e é estimulada pelo SUS, com os objetivos de proporcionar celeridade no processo de alta hospitalar com cuidado continuado no domicílio, minimizar intercorrências clínicas, diminuir os riscos de infecçóes hospitalares por longo tempo de permanência de pacientes no ambiente hospitalar e propor a autonomia para o paciente no cuidado fora do hospital (BRASIL, 2014b). A TND então tem como finalidade recuperar ou manter o estado de saúde, funcionalidade e comodidade do paciente, além de ser conveniente para reduzir os custos hospitalares (SBNPE, 2012).

A TND pode ser instituída em regime oral, enteral ou parenteral e é destinada para o tratamento clínico de pacientes de média e alta complexidade. É um método seguro e econômico, no quesito custo e benefício, e requer planejamento e monitoramento pela equipe especializada em terapia nutricional (DE LEGGE; IRETON-JONES, 2007).

A alimentação nutricional enteral domiciliar (TNED) causa efeitos físicos, psicológicos e sociais na vida dos pacientes e de seus cuidadores (DAY, 2017). Apesar de apresentar efeitos positivos na recuperação e/ou manutenção do estado nutricional dos indivíduos, a TND carece de estudos com maiores proporçôes que possam identificar tanto as condiçóes clínicas destes pacientes quanto os efeitos adversos que esta pode acarretar aos indivíduos. O objetivo desta revisão é detalhar os estudos em TNED no âmbito infanto-juvenil e descrever quais os diagnósticos clínicos mais comuns entre esses indivíduos e os possíveis efeitos colaterais que a TNED pode provocar nesses pacientes, além de detalhar a via acesso para a nutrição enteral mais recorrente e os tipos de dietas enterais utilizadas como aporte energético para suprir as necessidades calóricas destes pacientes.

\section{Método}

Trata-se de uma revisão integrativa de literatura, de acordo com método estabelecido por Whittemore e Knafl (2005), respeitando as fases estabelecidas para sua realização, como: formulaçáo da hipótese, busca na literatura conforme os critérios de inclusão e exclusão, categorização dos estudos e avaliação, interpretação dos resultados e elaboração da revisão. 
Estabeleceu-se como pergunta norteadora "Quais as condiçôes clínicas que determinam a indicação da TNED na população infanto-juvenil?”. Buscou-se investigar quais são os efeitos adversos que a terapia domiciliar pode acarretar ao indivíduo, qual a via de acesso mais recorrente para a administração da nutrição enteral e os tipos de dietas enterais mais utilizadas nesses pacientes.

A pesquisa de artigos foi conduzida no mês de maio de 2018 por meio de buscas nas bases de dados: Scientific Eletronic Library Online (SciElo) e US National Library of Medicine National Institutes of Health (PubMed), utilizando as seguintes palavras-chaves: 'home enteral nutrition', 'home tube feeding' e 'children'. Foram selecionados artigos nos idiomas: espanhol e inglês, publicados entre os anos de 2006 a 2018.

Os critérios de inclusão foram estudos indexados nas bases de dados envolvendo crianças e adolescentes do sexo masculino e feminino, com idade limite até 19 anos e pacientes em regime de suporte nutricional por via oral concomitante com a nutrição enteral. Os critérios exclusão foram estudos que não se enquadravam conforme a pergunta norteadora, aqueles que apresentaram dados inconsistentes, como estudos que retratavam a TNED para adultos e crianças, entretanto não apresentavam dados para caracterizar a populaçáo infanto-juvenil de forma distinta, estudos que abordavam a TNED para uma patologia específica, estudos com o intuito de avaliar a eficácia da nutrição enteral artesanal na TND, estudos relacionados a terapia nutricional parenteral domiciliar e estudos que retratavam de recomendaçóes para a utilização da TNED.

\section{Síntese de dados}

$\mathrm{Na}$ fase de categorização e avaliação dos estudos foram encontrados 379 artigos, sendo excluídos aqueles que não se adequavam aos critérios estabelecidos. Dezesseis estudos atenderam os critérios de inclusão e foram selecionados, sendo cinco encontrados no SciElo e onze na PubMed. Em relação ao idioma houve o predomínio de estudos na língua inglesa $(\mathrm{n}=11)$ e a minoria em língua espanhola $(\mathrm{n}=5)$. A Figura 1 apresenta os resultados detalhados durante a seleção dos artigos e a Tabela 1 demonstra um resumo dos achados mais relevantes reportados em cada estudo incluído. 
Figura 1. Fluxograma da seleção de artigos

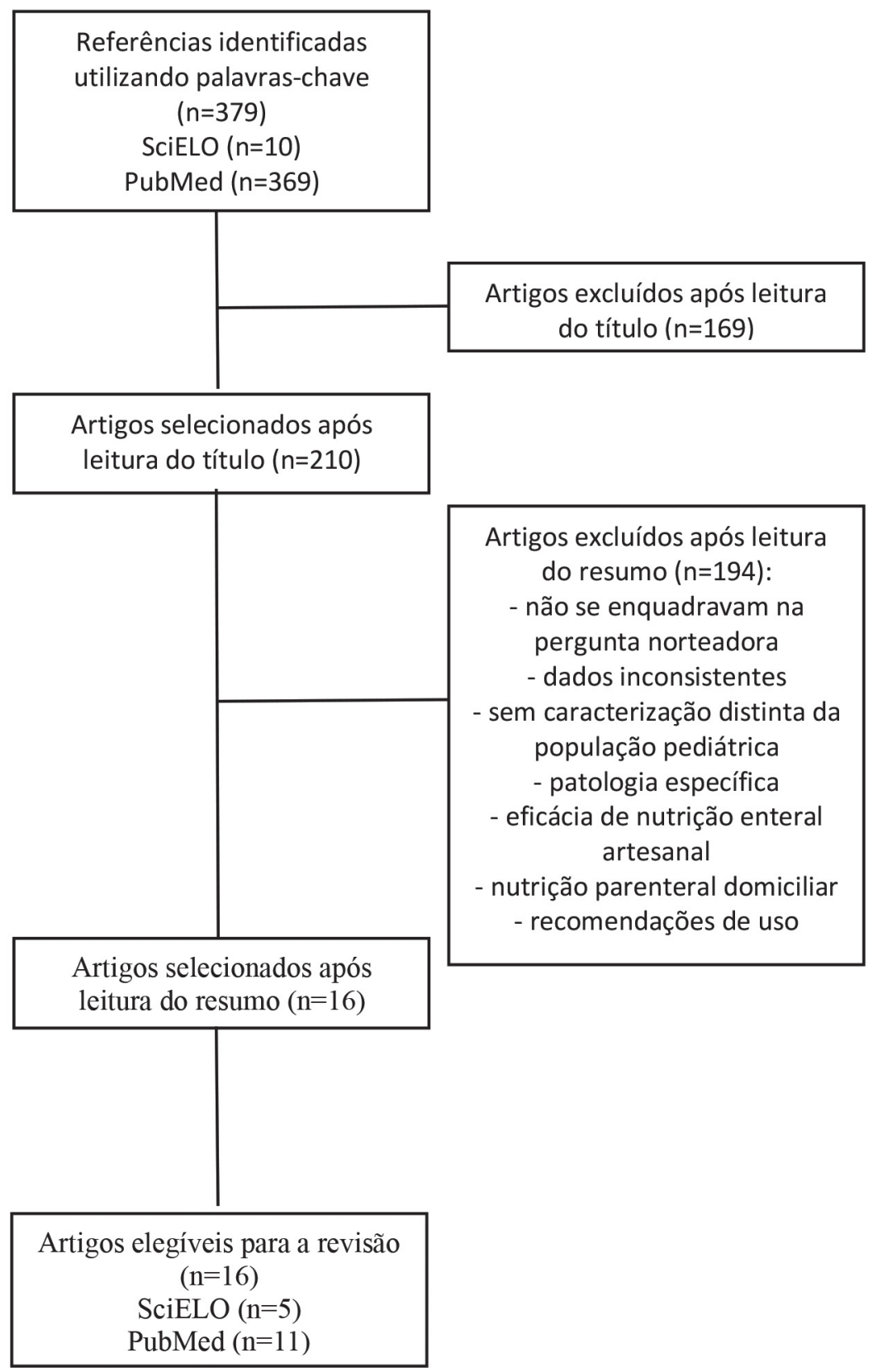


Tabela 1. Diagnósticos clínicos comuns nos pacientes infanto-juvenis em TNED

\begin{tabular}{|c|c|c|c|c|}
\hline Autor/Ano & País & Amostra avaliada & Resultados & Conclusão \\
\hline $\begin{array}{l}\text { Zaban et al. } \\
2010\end{array}$ & Brasil & $\begin{array}{l}184 \text { pacientes } \\
\text { menores de } 18 \\
\text { anos (referente ao } \\
\text { ano de 2005) }\end{array}$ & $\begin{array}{l}\text { Doenças do trato } \\
\text { gastrointestinal e doenças } \\
\text { causadas pela má absorção e } \\
\text { digestão de nutrientes (doença } \\
\text { inflamatória intestinal, } \\
\text { síndrome do intestino curto, } \\
\text { diarreia severa, doença celíaca, } \\
\text { alergia a proteína do leite de } \\
\text { vaca, fibrose cística) doenças } \\
\text { neurológicas, malformaçôes } \\
\text { congênitas, doenças cardíacas, } \\
\text { doenças ocasionadas pela } \\
\text { prematuridade, doença renal e } \\
\text { falha do crescimento. }\end{array}$ & $\begin{array}{l}\text { O estudo preconiza } \\
\text { que a criança tenha a } \\
\text { alta hospitalar o quanto } \\
\text { mais cedo possível para } \\
\text { diminuir os riscos de } \\
\text { doenças oportunistas, e } \\
\text { possa receber o suporte } \\
\text { nutricional enteral em seu } \\
\text { domicílio considerado um } \\
\text { ambiente seguro. }\end{array}$ \\
\hline $\begin{array}{l}\text { Tinoco et al. } \\
2013\end{array}$ & Chile & $\begin{array}{l}37 \text { pacientes } \\
\text { pediátricos } \\
\text { (referente aos anos } \\
\text { de 2010-2011) }\end{array}$ & $\begin{array}{l}\text { Doenças neurológicas, doenças } \\
\text { cardiovasculares, doenças } \\
\text { neuromusculares, malformaçôes } \\
\text { congênitas e outras não } \\
\text { mencionadas. }\end{array}$ & $\begin{array}{l}\text { A TNED permite que } \\
\text { a criança continue sua } \\
\text { recuperaçáo no âmbito } \\
\text { domiciliar, onde ela tem } \\
\text { o apoio mais próximo dos } \\
\text { pais e parentes. }\end{array}$ \\
\hline $\begin{array}{l}\text { Giner et al. } \\
2012\end{array}$ & Espanha & $\begin{array}{l}304 \text { pacientes } \\
\text { menores de } 18 \\
\text { anos (referente } \\
\text { ao ano de } 1995 \text { a } \\
2004 \text { ) }\end{array}$ & $\begin{array}{l}\text { Neoplasias, doenças } \\
\text { neurológicas, doenças do trato } \\
\text { gastrointestinal e ocasionadas } \\
\text { pela má absorção e digestão } \\
\text { de nutrientes (doença } \\
\text { gastroesofagiana, fibrose } \\
\text { cística, doença celíaca, alergia } \\
\text { a proteína do leite de vaca, } \\
\text { doença inflamatória intestinal, } \\
\text { síndrome do intestino curto, } \\
\text { diarreia severa), insuficiência } \\
\text { renal e doenças neurológicas. }\end{array}$ & $\begin{array}{l}\text { Primeiro estudo da Espanha } \\
\text { que comtempla } 10 \text { anos } \\
\text { de acompanhamento de } \\
\text { pacientes pediátricos para } \\
\text { avaliar idade, indicaçấo } \\
\text { da TNED, via de acesso, } \\
\text { método de administração } \\
\text { da fórmula, tipo e fórmula } \\
\text { indicada. }\end{array}$ \\
\hline $\begin{array}{l}\text { Giner et al. } \\
2010\end{array}$ & Espanha & $\begin{array}{l}310 \text { pacientes } \\
\text { menores de } 14 \\
\text { anos (referente ao } \\
\text { ano de 2008) }\end{array}$ & $\begin{array}{l}\text { Doenças neurológicas, } \\
\text { neoplasias e outras } \\
\text { (hepatopatias, insuficiência } \\
\text { renal, síndromes de má } \\
\text { absorçáo). }\end{array}$ & $\begin{array}{l}\text { Aumento das enfermidades } \\
\text { e idade dos pacientes } \\
\text { necessitando se suporte } \\
\text { nutricional enteral }\end{array}$ \\
\hline $\begin{array}{l}\text { Berghe et al. } \\
2010\end{array}$ & Espanha & $\begin{array}{l}302 \text { pacientes } \\
\text { menores de } 14 \\
\text { anos (referente ao } \\
\text { ano de 2009) }\end{array}$ & $\begin{array}{l}\text { Doenças neurológicas, } \\
\text { neoplasias, síndrome de má } \\
\text { absorção, hepatopatia crônica, } \\
\text { insuficiência renal. }\end{array}$ & $\begin{array}{l}\text { O falecimento é a causa } \\
\text { mais frequente e a transição } \\
\text { da nutriçấo enteral para a } \\
\text { via oral. }\end{array}$ \\
\hline
\end{tabular}

continua... 


\begin{tabular}{|c|c|c|c|}
\hline Autor/Ano & País & Amostra avaliada & Resultados \\
\hline $\begin{array}{l}\text { Frias et al. } \\
2012\end{array}$ & Espanha & $\begin{array}{l}264 \text { pacientes } \\
\text { menores de } 14 \\
\text { anos (referente ao } \\
\text { ano de 2010) }\end{array}$ & $\begin{array}{l}\text { Doenças neurológicas, } \\
\text { neoplasias, síndrome de má } \\
\text { absorção severa, transtorno de } \\
\text { motilidade intestinal e estenose } \\
\text { esofágica não neoplásica. }\end{array}$ \\
\hline $\begin{array}{l}\text { Berghe et al. } \\
2014\end{array}$ & Espanha & $\begin{array}{l}62 \text { pacientes } \\
\text { pediátricos } \\
\text { (referente aos anos } \\
\text { de 2011-2012) }\end{array}$ & $\begin{array}{l}\text { Doenças neurológicas, câncer } \\
\text { de cabeça e pescoço, câncer } \\
\text { gastrointestinal, síndrome de má } \\
\text { absorção. }\end{array}$ \\
\hline $\begin{array}{l}\text { Berghe et al. } \\
2015\end{array}$ & Espanha & $\begin{array}{l}55 \text { pacientes } \\
\text { menores de } 14 \\
\text { anos (referente ao } \\
\text { ano de 2013) }\end{array}$ & $\begin{array}{l}\text { Doenças neurológicas, câncer } \\
\text { de cabeça e pescoço, câncer } \\
\text { gastrointestinal, síndrome de má } \\
\text { absorção, estenose esofágica não } \\
\text { neoplásica, disfagia severa. }\end{array}$ \\
\hline
\end{tabular}

Berghe et al. Espanha 144 pacientes 2017 pediátricos (referente aos anos Doenças neurológicas e disfagia 2014-2015) severa.

\section{Conclusão}

Determinação para

realizaçấo de gastrostomia em pacientes pediátricos que permanecerão em regime enteral $>8-12$ semanas.

Aumento de prescrição de TNED nos anos avaliados.

Melhorar a dinâmica de registros pelos centros de assistência, devido o crescimento de pacientes adultos e pediátricos em TNED a cada ano.

Atualizaçôes periódicas nos registros de pacientes para evitar os casos omissos e monitoramento do tempo que o paciente iniciou o suporte nutricional enteral.

$\begin{aligned} & \text { Daveluy et. França } \\ & 2006\end{aligned}$
$\begin{aligned} & 416 \text { pacientes } \\ & \text { pediátricos }\end{aligned}$

Doenças do trato gastrointestinal e síndromes (referente aos anos de 1990 a 2000)

279 questionários respondidos pelos

Krom et al. Holanda 2018 pais de crianças que estáo em TNED (referente ao ano de 2016) de má absorção (doença inflamatória intestinal, fibrose cística, síndrome do intestino curto, doença celíaca, alergia a proteína do leite de vaca, diarreia severa), doenças neurológicas e neuromusculares, câncer, falha no crescimento e outras doenças em menor recorrência.

Ingestấo alimentar inadequada, falha no crescimento ou desnutrição crônica, doenças metabólicas, acesso para administração de medicamentos, desordens digestivas e absortivas de nutrientes foram os principais motivos para indicação da nutrição enteral.
Entre os anos de 1990 a 2000 a indicação de TNED obteve um aumento significativo, salientando que a qualidade de vida destes pacientes deve ser investigada.

Os pais freqüentemente relatam efeitos colaterais e experiências negativas causadas pela nutrição enteral sugerindo um alto impacto na vida social dos pacientes e familiares.

continua... 


\begin{tabular}{|c|c|c|c|c|}
\hline Autor/Ano & País & Amostra avaliada & Resultados & Conclusão \\
\hline $\begin{array}{l}\text { Diamanti et } \\
\text { al. } 2006\end{array}$ & Itália & $\begin{array}{l}509 \text { pacientes } \\
\text { pediátricos } \\
\text { (referentes aos } \\
\text { anos de } 1988 \text { - } \\
2005 \text { ) }\end{array}$ & $\begin{array}{l}\text { Doenças do trato } \\
\text { gastrointestinal (síndrome } \\
\text { do intestino curto, } \\
\text { diarreia intratável, refluxo } \\
\text { gastroesofágico, atresia } \\
\text { biliar, atresia esofagiana, } \\
\text { doença de Crohn), doenças } \\
\text { neuromusculares, doenças } \\
\text { metabólicas, doenças } \\
\text { cardiovasculares, doença renal e } \\
\text { Síndrome da Imunodeficiência } \\
\text { Adquirida (AIDS). }\end{array}$ & $\begin{array}{l}\text { A TNED em pediatria é } \\
\text { segura e bem tolerada. }\end{array}$ \\
\hline $\begin{array}{l}\text { Diamanti et } \\
\text { al. } 2013\end{array}$ & Itália & $\begin{array}{l}757 \text { pacientes } \\
\text { pediátricos } \\
\text { (referente aos anos } \\
1996-2009 \text { ) }\end{array}$ & $\begin{array}{l}\text { Desnutrição, inadequada } \\
\text { ingestão de nutrientes, doenças } \\
\text { neurológicas, desordens } \\
\text { genéticas, doenças do trato } \\
\text { gastrointestinal, doenças } \\
\text { crônicas renais e pulmonares, } \\
\text { malformações cardíacas } \\
\text { congênitas, doenças hepáticas e } \\
\text { erros inatos do metabolismo. }\end{array}$ & $\begin{array}{l}\text { Aumento expressivo de } \\
\text { crianças em TNED, sendo } \\
\text { a principal patologia } \\
\text { referenciada as doenças } \\
\text { neurológicas, a TNED } \\
\text { apresenta efeitos adversos, } \\
\text { mas náo sấo as causas de } \\
\text { falecimentos. }\end{array}$ \\
\hline $\begin{array}{l}\text { Rosen et al. } \\
2014\end{array}$ & $\begin{array}{l}\text { Monte } \\
\text { Sinai } \\
\text { (Egito) }\end{array}$ & $\begin{array}{l}87 \text { pacientes } \\
\text { pediátricos } \\
\text { (referente aos anos } \\
\text { de 2010-2013) }\end{array}$ & $\begin{array}{l}\text { Doença cardíaca congênita, } \\
\text { doenças metabólicas, doenças } \\
\text { neurológicas, doença hepática, } \\
\text { prematuridade e doença } \\
\text { inflamatória intestinal. }\end{array}$ & $\begin{array}{l}\text { Pesquisas futuras e com } \\
\text { amostras de indivíduos } \\
\text { maiores são necessárias para } \\
\text { determinar se a TNED irá } \\
\text { causar algum impacto no } \\
\text { crescimento a longo prazo.. }\end{array}$ \\
\hline $\begin{array}{l}\text { Sidorkiewicz } \\
\text { et al. } 2012\end{array}$ & Polônia & $\begin{array}{l}525 \text { pacientes } \\
\text { pediátricos } \\
\text { (referente ao ano } \\
\text { de } 2010 \text { ) }\end{array}$ & $\begin{array}{l}\text { Doenças neurológicas, } \\
\text { desordens genéticas, doença } \\
\text { inflamatória intestinal, } \\
\text { síndrome do intestino } \\
\text { curto e outras doenças do } \\
\text { trato gastrointestinal não } \\
\text { mencionadas, fibrose cística, } \\
\text { insuficiência renal crônica, } \\
\text { neoplasias e desordens } \\
\text { metabólicas. }\end{array}$ & $\begin{array}{l}\text { Existe uma discrepância } \\
\text { em regiôes do país que } \\
\text { ofertam a TNED. Ainda } \\
\text { são escassos os centros } \\
\text { assistenciais em saúde em } \\
\text { algumas regiốes. }\end{array}$ \\
\hline $\begin{array}{l}\text { Klek et al. } \\
2014\end{array}$ & Polônia & $\begin{array}{l}142 \text { pacientes } \\
\text { pediátricos } \\
\text { (referente aos anos } \\
\text { de } 2007-2013 \text { ) }\end{array}$ & $\begin{array}{l}\text { A implementação da TNED } \\
\text { diminuiu as recorrências de } \\
\text { complicaçóes infecciosas, } \\
\text { pneumonia e infecção do trato } \\
\text { urinário. }\end{array}$ & $\begin{array}{l}\text { A implementação da } \\
\text { TNED acarreta em } \\
\text { diminuição dos gastos e } \\
\text { tempo de permanência } \\
\text { hospitalar para os pacientes. }\end{array}$ \\
\hline
\end{tabular}




\section{Terapia Nutricional Enteral Domiciliar (TNED) no Mundo}

Um estudo realizado no Brasil (ZABAN e NOVAES, 2010) apontou184 pacientes pediátricos em TNED, sendo 81 pacientes do sexo feminino e 103 do sexo masculino. Nos diagnósticos clínicos, prevaleceram as patologias como doenças do trato gastrointestinal e doenças causadas pela má absorção e digestão de nutrientes (doença inflamatória intestinal, síndrome do intestino curto, diarreia severa, doença celíaca, alergia a proteína do leite de vaca, fibrose cística) doenças neurológicas, malformaçóes congênitas, doenças cardíacas, doenças ocasionadas pela prematuridade, doença renal e falha do crescimento como indicativas para a administração da nutrição enteral no domicílio.

Segundo Tinoco et al. (2013), em estudo conduzido no Chile, foram coletados dados de prontuários no serviço de pediatria de um hospital entre os anos de 2010 a 2011 de 37 pacientes pediátricos aptos a TNED, e entre os diagnósticos clínicos mais prevalentes destacaram-se as doenças neurológicas, doenças cardiovasculares, doenças neuromusculares e malformaçōes congênitas.

A TNED na Espanha já vem sendo referenciada na literatura, enfatizando a consolidação de tal método fora do ambiente hospitalar. O primeiro estudo encontrado foi realizado por Giner et al. (2012) com 304 pacientes pediátricos, entre 1995 e 2004 . Entre os achados, 28\% dos pacientes eram menores de um ano de idade, e entre as condições clínicas para a indicação da nutrição enteral destacaram-se câncer, doenças do trato gastrointestinal e ocasionadas pela má absorção e digestão de nutrientes (doença gastroesofagiana, fibrose cística, doença celíaca, alergia a proteína do leite de vaca, doença inflamatória intestinal, síndrome do intestino curto, diarreia severa), insuficiência renal e doenças neurológicas. Os estudos realizados nos anos de 2008, 2009 e 2010 também na Espanha apontaram pacientes menores de 14 anos em TNED, sendo as doenças neurológicas, neoplasias, falha no crescimento, hepatopatia crônica, síndrome de má absorção severa e transtorno de motilidade intestinal, as principais patologias relatadas para a indicação da TNED (GINER et al., 2010; BERGHE et al., 2010; FRIAS et al., 2012).

Estudos subsequentes realizados na Espanha abrangeram a população adulta e infantil, com crescimento anual expressivo da TND pediátrica entre 2011 e 2015. Dentre as condiçôes clínicas reportadas para indicação, foram citadas as doenças 
neurológicas, neoplasias, síndrome de má absorção e disfagia grave (BERGHE et al., 2014; BERGHE et al., 2015; BERGHE et al., 2017).

Daveluy et al. (2006) realizaram estudo em um hospital universitário na França, com 416 pacientes pediátricos entre 1990 e 2000 aptos a TNED. Os resultados apontaram que os principais diagnósticos clínicos para a indicação foram doenças do trato gastrointestinal e síndromes de má absorção, doenças neurológicas e neuromusculares, câncer, falha no crescimento e outras doenças em menor recorrência.

Um estudo realizado na Holanda por Krom et al. (2018) registrou a prevalência de TND entre os anos de 2010 a 2014, sendo respectivamente 84-92-91-84-83 a cada 100 mil crianças e adolescentes. Entretanto, o principal propósito do estudo foi aplicar questionários aos parentes para investigar as principais causas para indicação da TNED no período que este foi conduzido (2016), sendo coletados 279 questionários e entre as causas destacam-se: ingestão alimentar inadequada, falha no crescimento ou desnutrição crônica, doenças metabólicas, via para administração de medicamentos e desordens digestivas e absortivas de nutrientes foram os principais motivos para indicação da nutrição enteral. Krom et al. (2018) ainda relatam efeitos adversos que a terapia nutricional pode resultar nas crianças como falta de apetite, vômitos, engasgos, náuseas e tosse.

Em estudo conduzido por Diamanti et al. (2006), foi retratada a TNED na Itália entre os anos de 1988 a 2005 em população de 509 pacientes pediátricos, tendo entre as causas para a indicação da TNED destacaram-se as doenças do trato gastrointestinal (síndrome do intestino curto, diarreia intratável, refluxo gastroesofágico, atresia biliar, atresia esofagiana, doença de Crohn), doenças neuromusculares, doenças metabólicas, doenças cardiovasculares, doença renal e Síndrome da Imunodeficiência Adquirida (AIDS). Outro estudo realizado por Diamanti et al. (2013) relatou 757 casos de crianças em TNED na Itália entre os anos de 1996 a 2009, sendo a indicação para tal conduta as seguintes condiçóes clinicas: desnutrição, inadequada ingestão de nutrientes, doenças neurológicas, desordens genéticas, doenças do trato gastrointestinal, doenças crônicas renais e pulmonares, malformaçóes cardíacas congênitas, doenças hepáticas e erros inatos do metabolismo. Em relação às complicaçôes da TND os pesquisadores citaram como recorrentes os quadros de vômitos, diarreia, pneumonia aspirativa, níveis glicêmicos e de eletrólitos descompensados e constipação intestinal. 
Um estudo de Rosen et al. (2016) foi conduzido em Monte Sinai (Egito), em uma amostra de 87 crianças os principais diagnósticos clínicos encontrados para a indicação de nutrição enteral foram doença cardíaca congênita, doenças metabólicas, doenças neurológicas, doença hepática, prematuridade e doença inflamatória intestinal.

Por meio do estudo de Sidorkiewicz et al. (2012) realizado na Polônia, foi demonstrado que o número de crianças em TNED cresceu de 433 a 525 de janeiro a dezembro de 2010 ( $\mathrm{n}=247$ meninas e 278 meninos), prevalecendo as doenças neurológicas como principal condição clínica, seguida de desordens genéticas, doença inflamatória intestinal, síndrome do intestino curto e outras doenças do trato gastrointestinal não mencionadas, fibrose cística, insuficiência renal crônica, neoplasias e desordens metabólicas. Klek et al. (2014) também conduziu um estudo com 456 pacientes adultos e crianças $(\mathrm{n}=142)$ em TNED na Polônia entre os anos de 2007 a 2013, e os resultados apontaram benefícios clínicos nesses antes e após a indicação da TNED com diminuição da prevalência de complicaçôes infecciosas, pneumonia e infecção do trato urinário.

\section{Vias de acesso da nutrição enteral}

A administração da dieta enteral pode ser realizada por meio da colocação de uma sonda na região nasal com posicionamento gástrico ou pós-pilórico, ou através de um acesso cirúrgico com alocação da sonda em posição gástrica (gastrostomia) ou jejunal (jejunostomia) (Baxter; Cecconello; Pinotti, 2013). Desta maneira, os estudos de TNED realizados na Espanha trazem, com o passar dos anos, uma diminuição da administração de fórmulas enterais pela via nasogástrica, aumentando as indicaçôes para ostomias. Segundo Giner et al. (2012), entre os anos de 1995 e 2004, houve uma maior porcentagem de crianças recebendo nutrição enteral pela via nasogástrica (71,7\%), quando comparado com a gastrostomia (27,7\%).

Nos estudos subsequentes, observa-se certa equivalência na utilização entre as vias, além de um caso em que foi mencionado a utilização da jejunostomia (BERGHE et al., 2014; BERGHE et al., 2015; BERGHE et al., 2017). Observou-se no estudo referente aos anos de 2011-2012 uma porcentagem de 43,5\% (nasogástrica), 33,9\% (gastrostomia) e 1,6\% (jejunostomia) (BERGHE et al., 2014). Já no estudo referente ao ano de 2013, houve maior recorrência de gastrostomia (51,0\%) (BERGHE et al., 2015), em relação ao ano de 2014 observou-se 52,0\% de crianças gastrostomizadas 
e em 2015 uma maior porcentagem dessas recebendo a nutrição enteral pela via nasogástrica $(50,8 \%)$ (BERGHE et al., 2017).

De acordo com os achados de Daveluy et al. (2006), a utilizaçáo de sonda nasogástrica em pacientes pediátricos era de 50,0\% até o ano de 1994, sendo que foi observado um decrescimento da utilização desta de 63,0\% para 35,0\% nos anos de 1990 a 2000, ademais as indicaçóes de gastrostomia tiveram seu aumento de 50,0\% para $61,0 \%$ neste mesmo período.

Ambos os estudos realizados na Itália demonstraram maior recorrência de sonda nasogástrica em detrimento da gastrostomia(DIAMANTI et al., 2006; DIAMANTI et al., 2013). Contudo, nos estudos de Sidorkiewicz et al. (2012), Tinoco et al. (2013) e Krom et al. (2018), observou-se uma maior frequência de gastrostomia em crianças, quando comparada com a utilização da sonda nasogástrica. Por outro lado, no estudo de Zaban e Novaes (2010), foi encontrada a mesma porcentagem de crianças com sonda nasogástrica e ostomizadas (gastrostomia).

Um fato importante a ser mencionado são os resultados apresentados por Krom et al. (2018), mostrando dados relacionados a substituição da sonda nasogástrica: $74(81,3 \%)$ dos responsáveis entrevistados referiram-se que a troca era realizada por uma equipe especializada, contudo foi relatado que a substituição da sonda trazia desconfortos sentimentais, destacando esse episódio como traumático e desagradável.

Tipos de fórmulas enterais utilizadas em TNED infanto-juvenil

As dietas enterais ou fórmulas enterais são composiçôes contendo nutrientes que vão garantir o aporte energético adequado ao paciente. Podem ser classificadas como polimérica, na qual os macronutrientes se apresentam na forma intacta; oligomérica, onde os macronutrientes se apresentam parcialmente hidrolisados e elementar, em que todos os macronutrientes se apresentam totalmente hidrolisados, ou seja, aptos a serem absorvidos pelo organismo (BAXTER; WAITZBERG, 2013).

Os estudos apresentam alguns tipos de fórmulas enterais utilizadas nos pacientes infanto-juvenil, sendo apontado por Zaban e Novaes (2010) uma maior porcentagem de dieta enteral polimérica pediátrica (44,9\%), seguida da dieta enteral com proteína hidrolisada (20,0\%), dieta enteral padrão (normocalórica) infantil (18,4\%), dieta enteral polimérica para adultos $(3,8 \%)$, dieta enteral para doenças específicas $(1,1 \%)$ e outras não relatadas (8,1\%). Já no estudo de Giner et al. (2012), foram observadas somente três variedades de dietas, sendo dieta enteral polimérica adulta e infantil (62,5\%) dieta enteral com proteína hidrolisada $(21,1 \%)$ e dieta enteral pediátrica $(16,4 \%)$. 
De acordo com os achados de Daveluy et al. (2006), a utilização de dieta polimérica pediátrica cresceu significativamente durante o período de estudo $(6,0 \%$ para 49,0\%). A utilização de dietas enterais com proteína hidrolisada não sofreu mudanças entre os anos de 1990 a 2000, entretanto, foram observadas o decréscimo na utilização de dietas enterais específicas para adultos no público infantil de 63,0\% até 1994 para 31,0\% no ano 2000.

No estudo de Diamanti et al. (2013), houve maior recorrência de utilização de dieta enteral com proteína do leite integral (padrão) (54,0\%), seguida de dietas enterais semi/elementares (33,0\%) e dietas enterais para doenças específicas $(13,0 \%)$. Já nos achados de Rosen et al. (2016), foi observada uma maior porcentagem na utilização de dieta enteral padrão (normocalórica) infantil (60,9\%), seguida da dieta enteral com proteína hidrolisada (10,3\%) e outros tipos distintos $(28,8 \%)$.

\section{Discussão}

Através da avaliação dos estudos, ficou evidente que a TND na Espanha foi pioneira, trazendo resultados anuais da prevalência de adultos e crianças recebendo nutrição enteral no domicílio. Por outro lado, temos a disseminação da TND em outros países europeus, como França, Itália, Polônia e Holanda, além de estudos realizados no Brasil, Chile e Monte Sinai (Egito). É de suma importância ressaltar que a TND é um método importante para o bem estar do paciente e que vem sofrendo algumas mudanças ao longo dos últimos anos e, devido a este fato, poucos estudos foram desenvolvidos na área.

Pode-se inferir que, apesar dos efeitos adversos que o tratamento domiciliar pode acarretar para o paciente infanto-juvenil, ainda assim é um fator protetor, pois, conforme menciona De Longueville et al. (2018), a desnutrição é uma doença atribuída a diversos fatores como má absorção e perda de nutrientes, aumento das necessidades energéticas ou ingestão alimentar reduzida, contudo, é uma doença recorrente em hospitais e pode provocar longos períodos de internaçóes gerando doenças mais graves. Sidorkiewicz et al. (2012) alegam as vantagens da TND sobre a internação hospitalar, onde a última provê maior custo ao paciente e irá colaborar para agravar o estado nutricional da criança.

Em relação às condiçôes clínicas relatadas das crianças, observou-se que grande parte delas são acometidas por doenças que requerem aporte nutricional pela via enteral a longo prazo. Sendo observado que a principal patologia reportada para a 
administração da TNED foram as doenças neurológicas. Ferreira et al. (2015) relatam que crianças com paralisia cerebral apresentam distúrbios ininterruptos na postura e no movimento que causa um prejuízo motor e gera impedimentos na realização das atividades de vida diária e consequentemente, uma dependência funcional. $\mathrm{O}$ estudo mencionado reforça que pacientes pediátricos com paralisia cerebral ou outra doença neurológica que acarrete prejuízo da capacidade de deglutição dos alimentos requerem suporte nutricional enteral devido à magnitude dos comprometimentos físicos que podem afetar diretamente a alimentação.

A grande maioria dos estudos citados referiu-se às doenças do trato gastrointestinal como patologias recorrentes em pacientes em TNED, entretanto, no estudo de Izaola et al. (2017), foi observado que a utilização de fórmula enteral de alta densidade foi capaz de diminuir a frequência dos sintomas gastrointestinais indesejáveis e ser mais tolerada pelos pacientes.

Considerando as vias de acesso para administração da nutrição enteral nos estudos citados, foi observada certa equivalência na utilização das duas vias (via nasogástrica e gastrostomia) nesses pacientes pediátricos (ZABAN; NOVAES, 2010; GINER et al., 2012; DAVELUY et al., 2006). A literatura apresenta que a TNED a longo prazo é marcada pela indicação de ostomias (gastrostomia, jejunostomia, jejunostomia transgástrica, gastrostomia endoscópica percutânea) para o conforto da criança, além disso, os pais e/ou responsáveis relataram que apesar do cuidado especial com a higienização, as ostomias foram benéficas para seus filhos e eles recomendam fortemente para outros pacientes (SHORTALL et al., 2015). Lyman et al. (2017) destacam ainda que a gastrostomia é a via de acesso mais indicada para crianças que irão permanecer em terapia nutricional por um longo período, sendo a sonda nasogástrica indicada para curto período.

Alguns estudos reportaram os tipos de dietas enterais utilizadas como suprimento energético para os pacientes em TNED, sendo administradas fórmulas que melhor se adequem à magnitude da doença, portanto não foi observada uma dieta enteral que seja padrão ouro a ser utilizada nesses pacientes. Observou-se que os tipos de dietas enterais relatadas são industrializadas, o que, segundo Gonçalves et al. (2018), apresenta alto custo econômico comparadas às dietas artesanais, porém oferecem praticidade, são completas nutricionalmente e mais seguras em relação ao aspecto microbiológico e à composição centesimal. Além disso, pacientes em TNED a longo prazo são susceptíveis a episódios de diarreia e constipação, sendo importante a 
utilização de dietas enterais adicionadas de fibras para minimizar esses desconfortos gastrointestinais (BENTO; GARCIA; JORDÁO JUNIOR, 2017).

Apenas os estudos de Krom et al. (2018) e Diamanti et al. (2013) apontarem efeitos adversos que a TNED pode acarretar nos pacientes, como falta de apetite, vômitos, engasgos, náuseas, tosse, diarreia pneumonia aspirativa, níveis glicêmicos e de eletrólitos descompensados, e constipação intestinal. Cutchma et al. (2016) constataram que as principais complicações decorrentes nesses pacientes foram náuseas $(11,9 \%)$, constipação intestinal $(11,9 \%)$, vômito $(9,5 \%)$, diarreia $(7,14 \%)$, obstrução da sonda (4,7\%), migração da sonda (4,7\%), pneumonia comunitária ou aspirativa $(2,3 \%)$ e broncoaspiração $(2,3 \%)$. Entretanto, a diarreia é um o efeito adverso mais comum (OLIVEIRA; PONTES; ROSA, 2018).

Apesar dos efeitos adversos a TNED, Klek et al. (2015) apontam vários efeitos benéficos para os pacientes como: melhora da condição clínica e reabilitação acelerada, rapidez na cicatrização de úlcera por pressão, redução de doenças respiratórias, redução de reinternaçóes e longos períodos de permanência hospitalares e prevenção de desnutrição.

A TNED proporciona benefícios para pacientes infanto-juvenis que necessitam de suporte nutricional contínuo, e uma vez que houve crescimento de pacientes em TNED, foram estipuladas recomendaçóes, entre elas, a apuração da melhor via de acesso para administração da dieta, condições clínicas compatíveis para tal método, idade mínima para iniciar a terapia nutricional, visto que se trata de uma população com necessidades especiais de cuidado para manutenção da saúde (SEVILLA; MCELHANON, 2016).

Planos assistenciais em TND tem sido desenvolvido por vários países, entretanto, muitos desses ainda carecem de melhorias e melhor planejamento para atender os pacientes, além disso, os profissionais devem estar aptos para atender a demanda e fornecer orientações aos pais (BAXTER; WAITZBERG, 2013).

\section{Conclusão}

Apesar dos achados envolvendo TNED se apresentarem ainda escassos, os estudos encontrados puderam demonstrar os benefícios e problemas principais decorrentes desse método de tratamento, sendo constatado que o tratamento nutricional em domicílio tem se difundido rapidamente no mundo. As principais condiçôes clínicas 
infanto-juvenis que propiciam a indicação da TNED são as doenças neurológicas, doenças do trato gastrointestinal e neoplasias. Entre os efeitos adversos destacaramse os vômitos, engasgos, tosse, diarreia e constipação intestinal. Observou-se que a administração da dieta ocorre tanto pela via nasogástrica quanto por gastrostomia, embora as ostomias tenham ganhado relevância. Vale ressaltar que as dietas infundidas nesses pacientes são industrializadas, oferecendo uma nutrição completa e protegendo o paciente de contaminaçóes microbiológicas.

A TNED é um meio viável para prevenir desnutrição hospitalar, tempo de permanência, reinternaçóes e redução dos custos hospitalares, além de trazer maior conforto para o paciente infanto-juvenil por estar mais próximo dos familiares.

\section{Referências}

BAXTER, Y. C.; CECCONELlO, I.; PINOTTI, H. W. Nutrição enteral domiciliar: introduçáo e bases técnicas. In: SILVA, S. M. C. S.; MURA, J. D. P. Tratado de alimentação, nutrição e dietoterapia. 2. ed. Sáo Paulo: Roca, 2013. p. 1035-1040.

BAXTER, Y. C.; WAITZBERG, D. L. Alimentação Enteral. In: SILVA, S. M. C. S.; MURA, J. D. P. Tratado de alimentação, nutrição e dietoterapia. 2. ed. São Paulo: Roca, 2013. p. 1015-1024. BENTO A. P. L.; GARCIA, R. W. D.; JORDÁO JUNIOR, A. A. Blenderized feeding formulas with nutritious and inexpensive foods. Revista de Nutrição, v. 30, n. 4, p. 525-534, 2017.

BERGHE C. W. et al. Registro espańol de nutrición enteral domiciliaria del año 2009; Grupo NADYA-SENPE. Nutrición Hospitalaria, v. 25, n. 6, p. 959-963, 2010.

BERGHE, C. W, et al. Registro del Grupo NADYA-SENPE de nutrición enteral domiciliaria en España, años 2014 y 2015. Nutrición Hospitalaria, v. 34, n. 1, p. 15-18, 2017.

BERGHE, C. W. et al. A home enteral nutrition (HEN); Spanish registry of NADYA-SENPE group; for the year 2013. Nutrición Hospitalaria, v. 31, n. 6, p. 2518-2522, 2015.

BERGHE, C. W. et al. Home enteral nutrition in Spain; NADYA registry 2011-2012. Nutrición Hospitalaria, v. 29, n. 6, p. 1339-1344, 2014.

BRASIL. Ministério da Saúde. Organização Pan-Americana da Saúde. Atenção domiciliar no SUS: resultados do laboratório de inovação em atenção. Brasília: Ministério da Saúde, 2014a.

BRASIL. Ministério da Saúde. Secretaria de Atenção à Saúde. Departamento de Atenção Básica. Departamento de Atenção Hospitalar e de Urgência. Manual de monitoramento e avaliação: Programa Melhor em Casa. Brasília: Ministério da Saúde, 2016.

BRASIL. Ministério da Saúde. Secretaria de Atenção à Saúde. Departamento de Atenção Básica. Política Nacional de Alimentação e Nutrição. Brasília: Ministério da Saúde, 2013. 
BRASIL. Ministério da Saúde. Secretaria de Atenção à Saúde. Departamento de Atenção Básica. Caderno de Atenção Domiciliar: Cuidados em Terapia Nutricional. Brasília: Ministério da Saúde, 2014b.

CUTCHMA, G. et al. Formulas alimentares: influência no estado nutricional, condição clínica e complicaçóes na terapia nutricional domiciliar. Nutrición Clínica y Dietética Hospitalaria, v. 6, n. 2, p. 45-54, 2016.

DAVELUY, W. et al. Dramatic changes in home-based enteral nutrition practices in children during an 11-year period. Journal of Pediatric Gastroenterology and Nutrition, v. 43, n. 1, p. 240-244, 2006.

DAY, T. Home enteral feeding and its impact on quality of life. British Journal of Community Nursing, v. 22, n.1, p. 14-16, 2017.

DE LEGGE, M. H.; IRETON-JONES, C. Home care. In: GOTTSCHLICH, M. M.; DE LEGGE, M. H,; MATTOX, T.; MUELLER, C.; WORTHINGTON, P. The ASPEN nutrition support core curriculum: a case-based approach - the adult patient. Silver Spring: American Society for Parenteral and Enteral Nutrition, 2007.

DE LONGUEVILLE, C. et al. Evaluation of nutritional care of hospitalized children in a tertiary pediatric hospital. Clinical Nutrition, v. 25, n. 1, p. 157-166, 2018.

DIAMANTI, A. et al. Home enteral nutrition in children: a 14-year multicenter survey. European Journal of Clinical Nutrition, v. 67, n. 1, p. 53-57, 2013.

DIAMANTI, A. et al. Home enteral nutrition in children: a 17-year period experience. Clinical Nutrition, v. 25, n. 1, p. 173-174, 2006.

FERREIRA, M. C. et al. Avaliação do índice de sobrecarga de cuidadores primários de crianças com paralisia cerebral e sua relação com a qualidade de vida e aspectos socioeconômicos. Revista Acta Fisiátrica, v. 22, n. 1, p. 9-13, 2015.

FRÍAS, L. et al. Nutrición enteral domiciliaria en España: registro NADYA del año 2010. Nutrición Hospitalaria, v. 27, n. 1, p. 266-269, 2012.

GINER, C. P. et al. Home enteral nutrition in children; a 10 year experience with 304 pediatric patients. Nutrición Hospitalaria, v, 27, n. 5, p. 1444-1450, 2012.

GINER, C. P. et al. Registro del Grupo NADYA-SENPE de Nutrición Enteral Domiciliaria en España en el año 2008. Nutrición Hospitalaria, v. 25, n. 5, p. 725-729, 2010.

GONÇALVES, E. S. et al. Bioethics and the human right to adequate feeding during enteral nutritional therapy. Revista Bioética, v. 26, n. 2, p. 260-270, 2018.

IZAOLA, O. et al. Estudio de tolerancia y aceptación de una fórmula enteral con alta densidad energética en pacientes de una unidad de coronarias. Nutritión Hospitalaria, v. 34, n. 1, p. 30-34, 2017. 
KLEK, S. et al. Home enteral nutrition reduces complications, length of stay, and health care costs: results from a multicenter study. The American Journal of Clinical Nutrition, v. 100, n. 2, p. 609-615, 2014.

KLEK, S. et al. The evolution of home enteral nutrition (HEN) in Poland during five years after implementation: a multicentre study. Nutritión Hospitalaria, v. 32, n. 1, p. 196-201, 2015. KROM, H. et al. Prevalence and side effects of pediatric home tube feeding. Clinical Nutrition, v. 37, n. 1, p. 1-6, 2018.

LYMAN, B. et al. Use of nasogastric feeding tubes for children at home: a template for caregiver education. Nutrition in Clinical Practice, v. 32, n. 6, p. 831-833, 2017.

OliveIRA. B. A. S.; PONTES, E. R. J. C.; ROSA, T. C. A. Resolution of control and monitoring instrument of nutritional therapy in the intensive care unit of a university hospital. Nutritión Hospitalaria, v. 35, n. 1, p. 19-24, 2018.

PIMENTA, F. S. et al. Agreement between Subjective Global Nutritional Assessment and the nutritional assessment of the World Health Organization. Jornal de Pediatria, v. 94, n. 6, p. 602-608, 2018.

RIBAS, D. F. et al. Nutrition assessment - Home-based nutritional therapy. Revista da Associação Médica Brasileira, v. 62, n. 8, p. 711-717, 2016.

ROSEN, D. et al. Home nasogastric feeds: feeding status and growth outcomes in a pediatric population. Journal of Parenteral and Enteral Nutrition, v. 40, n. 3, p. 350-354, 2016.

SEVILLA, W. M. A.; MCELHANON, B. Optimizing transition to home enteral nutrition for pediatric patients. Nutrition in Clinical Practice, v. 31, n. 6, p. 762-768, 2016.

SHORTALL, C. et al. Hospital to home paediatric enteral nutrition: parents need support. Irish Medical Journal, v. 108, n. 2, p. 46-48, 2015.

SIDORKIEWICZ, A. S. et al. Home enteral nutrition in children - 2010 nationwide survey of the polish society for clinical nutrition of children. European Journal of Pediatrics, v. 171, n. 1, p. 719-723, 2012.

SOCIEDADE BRASILEIRA DE NUTRIÇÃO PARENTERAL E ENTERAL - SBNPE. Terapia nutricional domiciliar. Revista da Associação Médica Brasileira, v. 58, n. 4, p. 408 411, 2012.

TINOCO, A. C. et al. Evaluación clínica del uso de nutrición enteral domiciliaria en pacientes pediátricos. Revista Chilena de Nutrición, v. 40, n. 1, p. 21-25, 2013

WAITZBERG, D. L. Nutrição oral, enteral e parenteral na prática clinica. 4. ed. São Paulo: Atheneu, 2009. 
WHITTEMORE, R.; KNAFL, K. The integrative review: updated methodology. Journal of Advanced Nursing, v. 52, n. 5, p. 546-553, 2005.

ZABAN, A. L. R. S.; NOVAES, M. R. C. G. Home enteral nutrition in children: a one-year experience with 184 patients in Distrito Federal, Brazil. Experimental Biology and Medicine, v. 235, n. 1, p. 584-589, 2010. 


\section{Abstract}

\section{Clinics aspects in children and youth patients in home enteral nutrition: an integrative review}

Home Enteral Nutrition is an effective method to ensure the nutritional compliance of an individual at home. The aim of this study was investigate the clinical aspects commonly recurrent in children and youth patients in home enteral nutrition. Research of articles was carried out through the databases: Scientific Electronic Library Online (SciElo) and US National Library of Medicine National Institutes of Health (PubMed) using the following keywords: 'home enteral nutrition', 'home tube feeding' and 'children'. Sixteen studies were selected in countries as Brazil, Spain, France, Netherlands, Italy, Poland and Monte Sinai, in which it was presented evidence that the main clinical conditions related to enteral nutritional therapy are neurological diseases, neoplasia in general and the upper and lower gastrointestinal tract diseases. Only two studies had reported the negative aspects of home therapy as episodes of gagging, coughing, vomiting, diarrhea, and constipation. Thus, through the clinical conditions reported, it is observed that the indication of home enteral nutrition is essential for these patients, which can prevent them from hospital malnutrition, decrease length of stay, rehospitalizations and reduce hospital costs, as well as bring greater comfort to patients by being closer to their family.

> Keywords: Nutritional therapy; Enteral nutrition; House calls; Children and youth; Child health. 